\title{
Pressure-Driven Evaporative Cooling in Atom Guides
}

\author{
Spencer E. Olson, ${ }^{1}$ Georg Raithel, ${ }^{2}$ and Andrew J. Christlieb ${ }^{3}$ \\ ${ }^{1}$ Air Force Research Laboratory, Space Vehicles Directorate, \\ 3550 Aberdeen Ave. SE, Kirtland Air Force Base, NM 87117-5776 \\ ${ }^{2}$ Physics Department, University of Michigan \\ 2477 Randall Lab, 450 Church Street, Ann Arbor, Michigan 48109-1120 \\ ${ }^{3}$ Mathematics Department, Michigan State University \\ D304 Wells Hall, East Lansing, Michigan 48824-1027
}

(Dated: June 28, 2021)

\begin{abstract}
We study steady-state evaporation in an atom guide via Monte Carlo simulations. The evaporation surface follows a specific profile as a function of longitudinal guide location. We demonstrate that the choice of evaporation profile significantly impacts the performance of the evaporation. Our simulations also demonstrate a significant performance boost in the evaporation when using a longitudinally compressed guide. We show that for a purely pressure-driven atom beam, it should be possible to reach degeneracy within a $0.5 \mathrm{~m}$ guide for experimentally feasible, albeit challenging, loading conditions.
\end{abstract}

PACS numbers: $64.70 . \mathrm{fm}$, 03.75.Pp

Keywords: Guided atom beam, Evaporative cooling, Evaporation surface, DSMC

The past several years have seen significant research to develop a continuous-wave (CW) atom laser [1]. Analogous to the impacts the CW optical laser had on precision measurements, a $\mathrm{CW}$ atom laser is expected to impact precision atom-based metrology via longer coherence lengths and greater continuity of temporal measurement coverage. A CW laser is identified by a continuously loaded and leaked macroscopic occupation of a quantum wave-function. The macroscopic-occupation state for an atom laser is a Bose-Einstein Condensate (BEC), where ultra-cold atoms can condense into the single ground state of a reservoir trap. Thus, in order for a CW atom laser to be established, atoms must be continuously cooled to sub-microkelvin temperatures, transported, and loaded into the BEC.

One of the primary tools for obtaining sub-microkelvin temperatures in atomic systems entails the use of forced evaporative cooling [2]. Evaporative cooling consists of removing the most energetic particles of a system, thereby lowering the total energy of the system. With forced evaporative cooling, the evaporation threshold is strategically lowered in order to maintain the energyremoval rate. As thermal energy is removed from an ensemble of atoms, the first-order coherence within the sample increases, often increasing the signal-to-noise ratio and precision of atomic measurements. This technique has been key in the forming of the Bose-Einstein condensate state of matter and has proven useful in many other cold-atom applications..

Some approaches for developing a $\mathrm{CW}$ atom laser attempt to establish a steady-state evaporative cooling process along the longitudinal direction of a guided cold atom beam. In this manner, an atomic beam is transferred into and transported by a magnetic guide [3] where the beam is cooled as it travels through the guide [4]. Beam temperature is lowered using an evaporation threshold that varies as a function of longitudinal

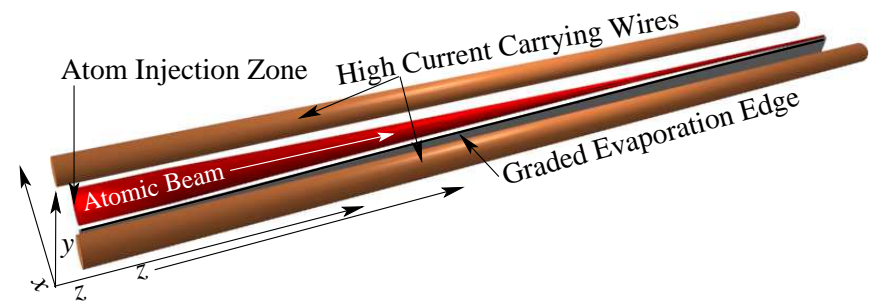

FIG. 1. Continuous evaporation is distributed in space and maintained in time by continually narrowing an effective evaporative surface in towards the center of the atom beam.

position. Fig. 1 shows a conceptual picture of distributed evaporative cooling. An atomic beam, injected at $z=0$, travels down the length of a magnetic guide. During this travel, an evaporation edge gradually removes more and more atoms from the beam. By maintaining collisionality throughout the guide, the beam is locally thermally equilibrated at all longitudinal positions, resulting in a continual decrease of the beam temperature $[5,6]$.

The goal of this paper is to demonstrate that a realistic and efficient strategy exists for establishing steady-state forced evaporation along the length of an atom beam. While the use of a supersonic beam of cold atoms is usually justified in order to inhibit thermal shortcuts between longitudinally distant atoms, we demonstrate that a pressure-driven flow has unique advantage for establishing steady-state cooling: not only are thermal shortcuts eliminated via collisionally viscous flow, but evaporative processes can be tuned within significantly shorter distances. We study a basic set of longitudinally varying evaporation surfaces that might be applied in an experiment. Simulation results are presented to depict the relative performance among the various strategies, and a clear relative optimum is demonstrated.

By employing a method known as Direct Simulation 
Monte Carlo (DSMC) [7], we simulate the evaporative cooling process in the magnetic guide. Simulations offer a reliable preview of the performance of a particular evaporation design. The DSMC method has been used successfully to simulate cold-atom collision processes such as evaporative cooling $[5,8]$ and $\mathrm{s} / \mathrm{d}$-wave collisions statistics [9]. In this work, we use a new gridless DSMC algorithm that provides uniform accuracy independent of density modulations. Our algorithm, the advantages thereof, and thorough testing are more thoroughly discussed in Ref. [10]. Simulations of the atom guide presented here were done using a parallel algorithm discussed in Ref. [11].

There are two key ingredients for establishing an efficient temperature gradient along the length of an atom guide using evaporative cooling: rethermalization and thermal isolation. Rethermalization occurs as a locally disturbed gas undergoes collisions to reach local thermal equilibrium. Thermal isolation in a guide, on the other hand, pertains to disallowing direct energy exchange between longitudinally distant groups of atoms.

As an example of rethermalization, consider a uniform gas of ${ }^{87} \mathrm{Rb}$ atoms with initial conditions given by $n_{\mathrm{i}}=1.25 \times 10^{8} \mathrm{~cm}^{-3}$ and $T_{\mathrm{i}}=100 \mu \mathrm{K}$, where $n_{\mathrm{i}}$ and $T_{\mathrm{i}}$ are the initial number density and temperature of the sample respectively. The velocity distribution of this gas can be represented by a normal distribution as shown in the top dashed curve in Fig. 2. A disturbance is created

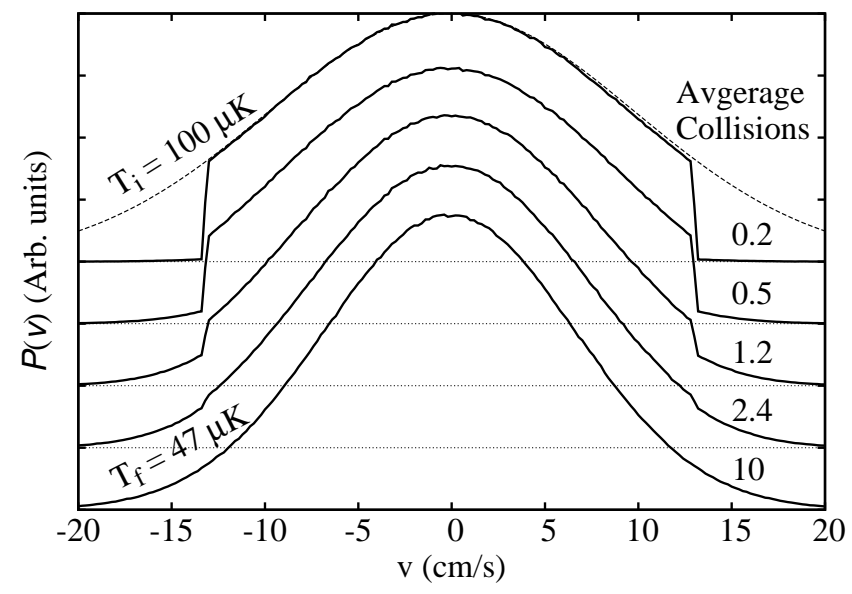

FIG. 2. The velocity distribution of a $100 \mu \mathrm{K}$ sample of ${ }^{87} \mathrm{Rb}$ atoms is truncated and allowed to rethermalize via collisions. Approximately 20\%, of the initial atom population, is removed as the wings of the thermal distribution are truncated. After roughly 10 collisions per atom, the velocity distribution errs, on average, less than $(1 / 100) \%$ from a thermal distribution with $T=47 \mu \mathrm{K}$.

by suddenly removing the $20 \%$ most energetic particles such that the velocity distribution is truncated as approximately represented by the highest solid curve in Fig. 2 . As shown in Fig. 2, the rethermalization process begins immediately with the first collisions after the truncation. Each collision quickly brings the velocity distribution of the gas closer and closer to a Boltmann distribution until the sample reaches thermal equilibrium. Because energy was removed via the truncation, the resulting Boltzmann distribution is narrower and more peaked, representing a colder collection of atoms. Depending on the final error admissible, Fig. 2 shows that the rethermalization time is in the range of $2-10 \gamma_{\mathrm{coll}^{-1}}{ }^{-1}$ where $\gamma_{\mathrm{coll}}$ is the average collision rate $[8,12,13]$.

Thermal isolation in the guide direction, $z$, is critical to ensure that hotter, upstream atoms cannot reach, collide with, and heat downstream portions of the atomic beam. Problematic longitudinal heat conduction arises from (1) low stream densities that result in collisionless flow or (2) high-angular-momentum trajectories of beam atoms through the guide [14]. Avoiding heat conduction is made possible by first maintaining collisionality and second, removing atoms that reach large guiding radii. High collisionality ensures that atoms exchange momentum only with other atoms of similar kinetic energy in nearly the same portion of the beam. By choosing an injection velocity $v_{\|_{0}}$ on the same order as the initial thermal velocity, flow is pressure driven instead of being primarily due to the inertia of the incident flow. Evaporative removal of all atoms outside of a $z$-dependent, critical radius ensures that high-angular-momentum states are disallowed.

Assuming that a particular temperature gradient can be established, the goal becomes to identify the type of gradient which results in the highest final phase-space density $n \lambda_{\text {th }}^{3}$ where $n$ is the number density and $\lambda_{\text {th }}$ is the thermal de Broglie wavelength given by

$$
\lambda_{\mathrm{th}}=\sqrt{\frac{2 \pi \hbar^{2}}{M k_{\mathrm{B}} T}} .
$$

Neglecting the longitudinal potential as a degree of freedom, each particle in the system has an average energy equal to $5 k_{\mathrm{B}} T / 2$. For simplicity, we assume that the edge of the near-thermal spatial distribution is at a transverse potential energy of $15 k_{\mathrm{B}} T / 2$. The exact value of this assumption is arbitrary but must result in a nearly total inclusion of the number and kinetic-energy distributions. For the choice of $15 k_{\mathrm{B}} T / 2,99.9 \%$ of the distribution with $99.5 \%$ of the kinetic energy lies within this range. By removing atoms that reach $U(\overrightarrow{\mathbf{x}})=15 k_{\mathrm{B}} T / 2+\Delta$, where $\Delta$ is the minimum energy in the center of the trap given by $\Delta=g_{F} m_{F} \mu_{B} B_{0}$, we can thus force the guide to support a temperature no greater than $T$. To establish a particular temperature gradient through the guide, we define the evaporation threshold function $\mathfrak{F}(z)$ as the potential energy $U(\overrightarrow{\mathbf{x}})$ at which atoms are removed at a longitudinal location $z$ in the guide. This results in an atomic distribution that increasingly narrows as the beam progresses down the guide. Fig. 3 shows such an atom distribution and the corresponding evaporation threshold surface that causes this narrowing, where $L$ is the length of the guide over which the evaporation threshold changes.

To evaluate the effect of the evaporation threshold function $\mathfrak{F}(z)$ on the final phase-space density $n \lambda_{\text {th }}^{3}$, we 


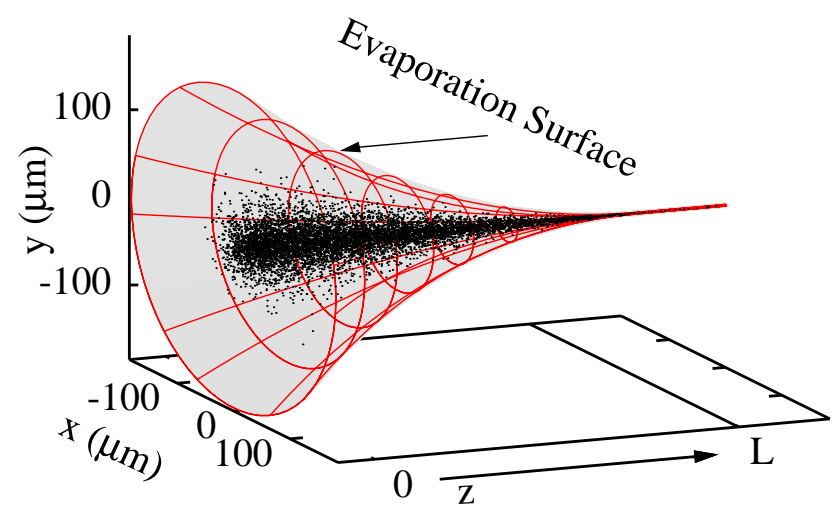

FIG. 3. Snapshot of the guided atom beam under the influence of forced evaporative cooling. The 3D surface indicates the location of the evaporation surface.

explore a (somewhat arbitrary) basic set given by:

$$
\mathfrak{F}(z) \sim\left\{\begin{array}{cc}
T_{\mathrm{f}}+\left(T_{\mathrm{i}}-T_{\mathrm{f}}\right) \cdot[1- & (z / L)]^{1 / 2}, \\
T_{\mathrm{i}}+\left(T_{\mathrm{f}}-T_{\mathrm{i}}\right) \cdot & (z / L)^{2}, \\
T_{\mathrm{i}}+\left(T_{\mathrm{f}}-T_{\mathrm{i}}\right) \cdot & (z / L), \\
T_{\mathrm{i}}+\left(T_{\mathrm{f}}-T_{\mathrm{i}}\right) \cdot & (z / L)^{1 / 2}, \\
T_{\mathrm{f}}+\left(T_{\mathrm{i}}-T_{\mathrm{f}}\right) \cdot[1- & (z / L)]^{2}
\end{array}\right\}, 0 \leq z \leq L
$$

Each of the basic $\mathfrak{F}(z)$ functions in Eq. 1, shown in Fig. 4, serves to evaluate a particular strategy for evaporative cooling. Each represents a different level of compromise between the need to establish thermal isolation (by removing high energy atoms earlier) and the need to achieve a high density in the downstream portions of the guide.

As the atoms travel down the guide, the evaporation process depletes the atom number and can eventually stall (on the timescale of the guide traversal) as the collision rate decreases. To prevent the forced evaporation from stalling, the atoms are magnetically squeezed to enhance the collision rate. In a two-wire magnetic guide, this is done by simply decreasing the separation between the two wires as a function of $z$. The computed magnetic field used for this work corresponds to two parallel currents $(I=150 \mathrm{~A})$ with a separation of $5.175 \mathrm{~mm}$ at $z=0$ linearly decreasing to $4.175 \mathrm{~mm}$ at $z=50 \mathrm{~cm}$. This separation results in gradients similar to the apparatus in Ref. [3]. Compression enhances the density and thus helps to maintain the collision rate as the atom number diminishes through the evaporative cooling process. Compression as a function of longitudinal position in the guide is analogous to temporal compression performed in standard BEC formation [13, 15]. It should be noted that the work done in Ref. [5] simulated evaporation in a non-compressed magnetic guide.

After forced evaporation ends at $z=L=40 \mathrm{~cm}$, the atoms continue until they reach an elastically reflecting barrier at $z=50 \mathrm{~cm}$. As described in [3], this wall could be created by a blue-detuned sheet of light, such that a

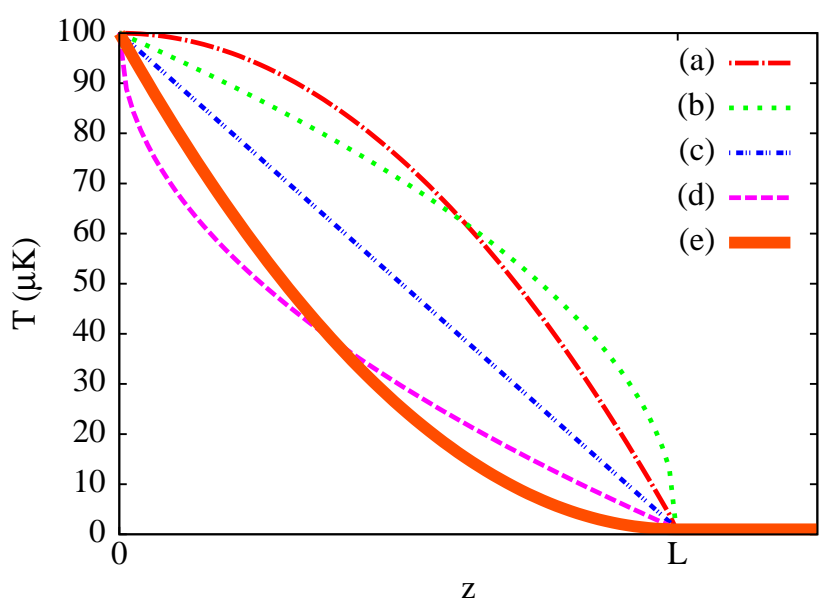

FIG. 4. Evaporation strategies of interest for this paper. The curves here depict the radial position $\rho$ at which atoms are removed from the system. By lowering the barrier in the forward direction, a forced evaporative cooling is imposed. The curves (a)-(e) represent the lines of $\mathfrak{F}(z)$ (Eq. 1), in order respectively. For $z \leq 0$ and $z \geq L, \mathfrak{F}(z)$ is held constant. These different strategies are to provide a set of basic types of evaporation surfaces that might be used experimentally.

three dimensional trap is established wherein a condensate can form.

To simulate the atom guide, ${ }^{87} \mathrm{Rb}$ atoms are injected at $z=0$ into an initially empty guide at a rate of $3 \times 10^{9} \mathrm{~s}^{-1} \Delta t$ per timestep $\Delta t$ with an average stream velocity of $v_{\|}=0$ and a temperature $T=100 \mu \mathrm{K}$. For these simulations, $L=40 \mathrm{~cm}$. As allowed by the Boltzmann equation, each simulated particle is scaled to represent $F_{\mathrm{N}} \geq 1$ rubidium atoms, such that each simulated particle has a cross section $F_{\mathrm{N}} \sigma_{\mathrm{T}}$ where $\sigma_{\mathrm{T}}$ is the total cross section of a single particle. Scaled representative particles decrease the computational burden and are typical in gas dynamics simulations. The figure of merit for choosing $F_{\mathrm{N}}$ is the ratio of the average distance between colliding particles to the mean free path [10]. A large $F_{\mathrm{N}}$ results in higher values of this ratio. If this ratio is too large $(\gtrsim 1)$, the collisionality of the simulation is significantly diminished. For this work, $F_{\mathrm{N}}$ was chosen as high as possible $\left(5 \times 10^{3}\right)$ without significantly diminishing the collision processes. It should be noted that a decrease in $F_{\mathrm{N}}$ will only result in a more collisional system and a larger temperature gradient along the length of the guide. These simulations therefore represent a lower bound of the evaporative-cooling performance.

By using a very low input stream velocity, it is expected that the pressure driven flow will allow a very short guide that is still able to maintain a strong temperature gradient. The input source is assumed to be in the $\left|F=2, m_{\mathrm{F}}=2\right\rangle$ stretched state. Thus, atoms are guided by a potential $U(\overrightarrow{\mathbf{x}})$ given by

$$
U(\overrightarrow{\mathbf{x}})=m g \overrightarrow{\mathbf{x}} \cdot \hat{\mathbf{y}}+\mu_{\mathrm{B}}\left|\overrightarrow{\mathbf{B}_{0}}+\overrightarrow{\mathbf{B}}(\overrightarrow{\mathbf{x}})\right|
$$




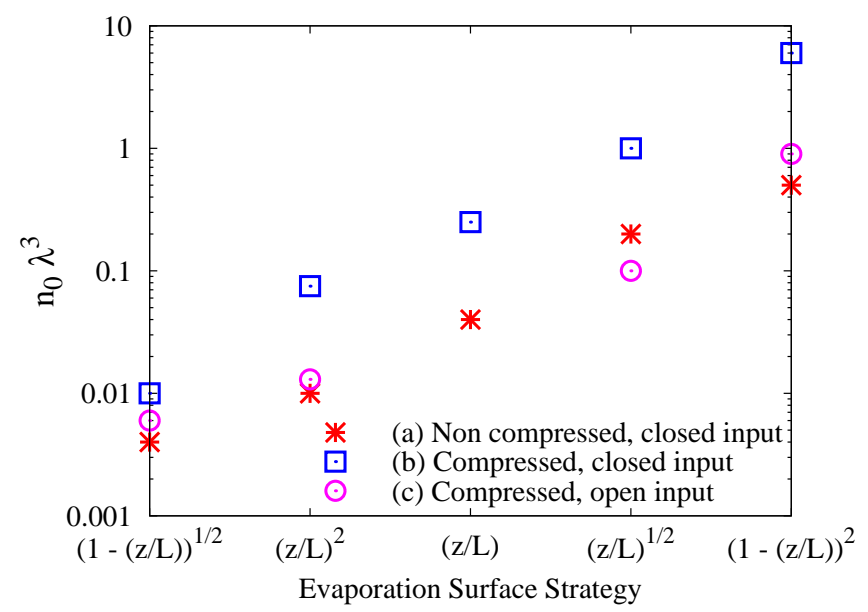

FIG. 5. Final phase space density $n \lambda_{\text {th }}^{3}$ of the different evaporation strategies. (a) Non-compressed guide has a constant magnetic field gradient of $750 \mathrm{G} / \mathrm{cm}$. A reflecting barrier is also placed at $-4 \mathrm{~cm}$. (b) Compressed guide begins with a gradient of $750 \mathrm{G} / \mathrm{cm}$ and ends with $1500 \mathrm{G} / \mathrm{cm}$. The reflecting barrier at $-4 \mathrm{~cm}$ is also present here. (c) Compressed guide $(750 \mathrm{G} / \mathrm{cm} \rightarrow 1500 \mathrm{G} / \mathrm{cm})$ without reflective barrier at $-4 \mathrm{~cm}$.

where $\overrightarrow{\mathbf{B}}(\overrightarrow{\mathbf{x}})$ is the magnetic field resulting from two parallel wire currents and $B_{0}$ is a small longitudinal bias $(0.5 \mathrm{G})$ to prevent non-adiabatic Majorana spin flips. The injected atom stream is mode-matched to the guide according to the distribution $\left.P(\overrightarrow{\mathbf{x}}, \overrightarrow{\mathbf{v}})\right|_{z=0}$ given by

$$
\left.P(\overrightarrow{\mathbf{x}}, \overrightarrow{\mathbf{v}})\right|_{z=0} \propto \exp \left[-\frac{M|\overrightarrow{\mathbf{v}}|^{2}}{2 k_{\mathrm{B}} T}-\frac{\left.U(\overrightarrow{\mathbf{x}})\right|_{z=0}}{k_{\mathrm{B}} T}\right] .
$$

Each timestep of the simulation is broken into two major sub-steps: collisionless motion followed by momentum exchanging collisions within nearest-neighbor atom groups, as described in Ref. [10].

To quantify the results of the simulated evaporative cooling, we examine the increase in $n \lambda_{\text {th }}^{3}$ over the length of the guide. For all cases, $n \lambda_{\mathrm{th}}^{3} \sim 10^{-4}$ at $z=0$. Fig. 5 compares the value of $n \lambda_{\text {th }}^{3}$ at $z=L$ for each instance of $\mathfrak{F}(z)$ in Eq. 1. For the results shown in Fig. 5, it is clear that the performance depends greatly on the form of
$\mathfrak{F}(z)$, with $\mathfrak{F}(z) \sim(1-(z / L))^{2}$ being the best candidate and $\mathfrak{F}(z) \sim(1-(z / L))^{1 / 2}$ being the least promising.

Fig. 5 also compares the following cases for each type of $\mathfrak{F}(z)$ : (a) a non-compressed guide with a wall at $-4 \mathrm{~cm}$, (b) a compressed guide with a wall at $-4 \mathrm{~cm}$, and (c) a compressed guide with no wall at $-4 \mathrm{~cm}$. For cases (a-b), the reflecting barrier at $-4 \mathrm{~cm}$ boosts the density throughout the guide and provides a best case scenario for comparison. This comparison shows that the compressed guide does indeed result in a higher final value of $n \lambda_{\mathrm{th}}^{3}$.

Our simulations show that an optimum strategy does exist for cooling atoms in a guide. By appropriately choosing the evaporation threshold function $\mathfrak{F}(z)$, it should be possible to achieve degeneracy even with short atom guides. Furthermore, as stated earlier, these simulations are expected to show only increased thermal isolation in the $z$ direction and hence greater evaporativecooling performance as the representative particle size $F_{\mathrm{N}} \rightarrow 1$. In addition, as $n \lambda_{\mathrm{th}}^{3} \rightarrow 1$, Bose statistics are expected to accelerate the condensation process of atoms into the ground state of the atom guide. We therefore conclude that a more accurate model of the physics would predict even higher evaporative cooling efficiency.

For high-precision metrology, one might use the evaporation profiles described here to obtain a steady-state (stationary in time) BEC at the end of the guide. For this, it is apparent that additional controls must be introduced for reducing the forward stream velocity. The most practical control variable for removing the longitudinal energy is the tilt applied to the guide, such that atoms are forced to climb a gravitational potential. Other methods of removing longitudinal energy could include moving magnetic [16-18] or perhaps optical potentials.

\section{ACKNOWLEDGMENTS}

This work was supported by the Naval Research Laboratory, Department of Defense High Performance Computing Modernization Program, and the Army Research Office (Project number 42791-PH). A. J. Christlieb was supported from AFOSR grants FA9550-11-1-0281, FA9550-12-1-0343.
[1] N. P. Robins, P. Altin, J. Debs, and J. Close, Physics Reports 529, 265 (2013).

[2] W. Ketterle and N. J. Van Druten, Adv. At. Mol. Opt. Phys. 37, 181 (1996).

[3] S. E. Olson, R. R. Mhaskar, and G. Raithel, Phys. Rev. A 73, 033622 (2006).

[4] T. Lahaye, Z. Wang, G. Reinaudi, S. P. Rath, J. Dalibard, and D. Guéry-Odelin, Phys. Rev. A 72, 33411 (2005).

[5] E. Mandonnet, A. Minguzzi, R. Dum,
I. Carusotto, Y. Castin, and J. Dalibard, Euro. Phys. J. D 10, 9 (2000).

[6] T. Lahaye and D. Gury-Odelin, Physical Review A 73, 063622 (2006).

[7] G. A. Bird, Molecular Gas Dynamics and the Direct Simulation of Gas Flows (Oxford University Press, New York, 1994).

[8] H. Wu and C. J. Foot, J. Phys. B 29, 321 (1996).

[9] A. C. J. Wade, D. Baillie, and P. B. Blakie, Phys. Rev. A 84, 023612 (2011). 
[10] S. E. Olson and A. J. Christlieb, J. Comp. Phys. 227, 8035 (2008).

[11] S. E. Olson, A. J. Christlieb, and F. K. Fatemi, Comp. Phys. Comm. 181, 2063 (2010).

[12] D. W. Snoke and J. P. Wolfe, Phys. Rev. B 39, 4030 (1989).

[13] C. R. Monroe, E. A. Cornell, C. A. Sackett, C. J. Myatt, and C. E. Wieman, Phys. Rev. Lett. 70, 414 (1993).

[14] R. Meppelink, R. van Rooij, J. Vogels, and P. van der Straten,
Phys. Rev. Lett. 103, 095301 (4 pp.) (2009/08/28).

[15] H. F. Hess, Phys. Rev. B 34, 3476 (1986).

[16] G. Reinaudi, Z. Wang, A. Couvert, T. Lahaye, and D. Guéry-Odelin, The European Physical Journal D-Atomic, Molecular, Optical and P

[17] H. L. Bethlem, Physics 1, 25 (2008).

[18] E. A. Imhof, "Chip-scale magnetic source of cold atoms," (2013). 